\title{
HOMOLOMORPHIC SECTIONAL CURVATURE OF SOME PSEUDOCONVEX DOMAINS
}

\author{
JEFFERY D. MCNEAL
}

(Communicated by Irwin Kra)

\begin{abstract}
The holomorphic sectional curvatures in the Bergman metric of a smooth bounded pseudoconvex domain in $\mathbf{C}^{2}$ are shown to be bounded in absolute value near a poinit of finite type in the boundary
\end{abstract}

\section{INTRODUCTION}

Several questions about the complex function theory of a domain in $\mathbf{C}^{n}$ have been explored by examining the curvature of the domain in an appropriate Hermitian metric. Various curvature conditions have been shown to characterize domains of holomorphy [10], extend the classical Schwarz lemma [8, 11], and insure the nondegeneracy of normal families of holomorphic mappings [5].

The abstract nature of many of the interesting metrics makes it difficult to obtain complete information about their curvature tensors except in some special cases. In [2], it was shown that for any bounded domain in $\mathbf{C}^{n}$, the holomorphic sectional curvature of the Bergman metric is less than 2. This result was extended to certain complex manifolds in [7]. Using Fefferman's asymptotic expansion for the Bergman kernel, Klembeck [6] showed that for a smooth bounded strongly pseudoconvex domain in $\mathbf{C}^{n}$, the holomorphic sectional curvatures of the Bergman metric approach $-4 /(n+1)$, that of the ball, near the boundary. For the domains $\Omega_{p}=\left\{\left|z_{1}\right|^{2}+\cdots+\left|z_{n-1}\right|^{2}+\left|z_{n}\right|^{2 p}<1\right\}, p>0$, Bland [3] has computed the curvature tensor of the Einstein-Kähler metric on $\Omega_{p}$ and shown that for $p \geq 1$, the sectional curvatures are bounded between negative constants.

In this paper, we estimate the sectional curvatures of the Bergman metric near the boundary of a smooth bounded pseudoconvex domain in $\mathbf{C}^{2}$. The result is

Theorem. Let $\Omega \subset \subset \mathbf{C}^{2}$ be a pseudoconvex domain with smooth boundary. If $z_{0} \in b \Omega$ is a point of finite type then there is a neighborhood $U$ of $z_{0}$ such

Received by the editors April 25, 1988 and, in revised form, July 11, 1988.

1980 Mathematics Subject Classification (1985 Revision). Primary 32A40, 32H10; Secondary 32A07, 32F20, 32H 15. 
that all the holomorphic sectional curvatures of the Bergman metric of $\Omega$ are bounded below by a negative constant in $U$.

The bound can be expressed in terms of the constant in the subelliptic estimate for the $\bar{\partial}$-Neumann problem.

\section{Preliminaries}

For a domain $\Omega$, denote by $A(\Omega)$ the set of holomorphic functions on $\Omega$. Let $X$ be a holomorphic tangent vector at a point $z \in \Omega$. The Bergman kernel $K_{\Omega}(z, \bar{z})$ and Bergman metric $B_{\Omega}(z ; X)$ for $\Omega$ are defined

$$
\begin{aligned}
& K_{\Omega}(z, \bar{z})=\sup \left\{|f(z)|^{2}: f \in A(\Omega),\|f\|_{L^{2}(\Omega)} \leq 1\right\}, \\
& B_{\Omega}(z ; X)=\frac{\sup \left\{|X f|: f \in A(\Omega), f(z)=0,\|f\|_{L^{2}(\Omega)} \leq 1\right\}}{(K(z, \bar{z}))^{1 / 2}} .
\end{aligned}
$$

Let $r(z)$ be a defining function for $\Omega$ and $z_{0} \in b \Omega$ be a point of finite type $m$. We may assume $\partial r / \partial z_{2} \neq 0(z)$ for $z \in U$, some neighborhood of $z_{0}$ and that $U$ is small enough so that all $z \in b \Omega \cap U$ are of type $\leq m$. It will be convenient to express the necessary estimates of the above quantities in terms of the special coordinate systems used in [4] and [9].

Proposition. For each $z^{\prime}=\left(z_{1}^{\prime}, z_{2}^{\prime}\right) \in U$, there exist numbers $d_{k}\left(z^{\prime}\right), k=$ $0, \ldots, m$, depending smoothly on $z^{\prime}$, such that $d_{0}\left(z^{\prime}\right) \neq 0$ and such that in the coordinates $\zeta=\left(\zeta_{1}, \zeta_{2}\right)$ defined by

$$
\left(z_{1}, z_{2}\right)=\Phi^{\prime}(\zeta)=\left(\Phi_{1}^{\prime}(\zeta), \Phi_{2}^{\prime}(\zeta)\right)
$$

where

$$
\begin{aligned}
& \Phi_{1}^{\prime}(\zeta)=z_{1}^{\prime}+\zeta, \\
& \Phi_{2}^{\prime}(\zeta)=z_{2}^{\prime}+d_{0}\left(z^{\prime}\right) \zeta_{2}+\sum_{k=1}^{m} d_{k}\left(z^{\prime}\right) \zeta_{1}^{k}
\end{aligned}
$$

the function $\rho$, given by $\rho(\zeta)=r\left(\Phi^{\prime}(\zeta)\right)$ satisfies

$$
\rho(\zeta)=r\left(z^{\prime}\right)+\operatorname{Re} \zeta_{2}+\sum_{\substack{j+k \leq m \\ j, k>0}} a_{j, k}\left(z^{\prime}\right) \zeta_{1}^{j} \bar{\zeta}_{1}^{k}+\mathscr{O}\left(\left|\zeta_{1}\right|^{m+1}+\left|\zeta_{2}\right||\zeta|\right) .
$$

Remark. The coordinates are canonical in the sense that only one biholomorphism of the form (1) normalizes the defining function to the form (2). Note that $z^{\prime}$ is mapped to the origin and that

$$
\left|J \Phi^{\prime}(\zeta)\right|=d_{0}\left(z^{\prime}\right) \neq 0, \quad \zeta \in \Phi^{\prime}(U)=V .
$$

where $|J(\cdot)|$ denotes the determinant of the holomorphic Jacobian matrix.

For $z^{\prime} \in U$, set $\Omega^{\prime}=\{\zeta: \rho(\zeta)<0\}$ and define

$$
A_{l}\left(z^{\prime}\right)=\max \left\{\left|a_{j, k}\left(z^{\prime}\right)\right|: j+k=l\right\}, l=2, \ldots, m,
$$


and for $\delta>0$,

$$
\tau\left(z^{\prime}, \delta\right)=\min \left\{\left(\delta / A_{l}\left(z^{\prime}\right)\right)^{1 / l}: 2 \leq l \leq m\right\} .
$$

The importance of the quantity $\tau\left(z^{\prime}, \delta\right)$ rests on the fact that

$$
R_{\delta}\left(z^{\prime}\right)=\left\{\zeta:\left|\zeta_{1}\right|<\tau\left(z^{\prime}, \delta\right),\left|\zeta_{2}\right|<\delta\right\}
$$

is essentially the largest polydisc about the origin on which $\rho$ changes by no more than $\delta$, see [9] for details. In the sequel, several quantities will be estimated in terms of powers of $\delta$ and $\tau\left(z^{\prime}, \delta\right)$ and it will be important that the inequalities depend on $z^{\prime}$ and $\delta$ only in the stated manner. To obviate the writing of useless constants, we use the notation $E \lesssim F$, for $E$ and $F$ functions which depend on $z^{\prime}$ and $\delta$, to mean that for some $c>0$, which is independent of $z^{\prime}$ and $\delta,|E| \leq c|F|$. We also write $E \approx F$ to mean that $E \lesssim F$ and $F \lesssim E$.

We now recall some estimates from [4] and [9] for the kernel function and Bergman metric for $\Omega^{\prime}$ along the normal ray at the origin. [4, Theorem 2] shows there exists a constant $b$, independent of $z^{\prime} \in U \cap b \Omega$ and small $\delta \in\left(0, \delta_{0}\right)$, so that for $\tilde{\zeta}=(0,-b \delta / 2)$

$$
K_{\Omega^{\prime}}\left(\widetilde{\zeta}, \widetilde{\zeta}^{-}\right) \approx \delta^{-2} \tau\left(z^{\prime}, \delta\right)^{-2}
$$

and

$$
B_{\Omega^{\prime}}(\widetilde{\zeta} ; Y) \approx\left|c_{1}\right| \tau\left(z^{\prime}, \delta\right)^{-1}+\left|c_{2}\right| \delta^{-1}
$$

if $Y=c_{1} \partial / \partial \zeta_{1}+c_{2} \partial / \partial \zeta_{2}$. [9, Theorem 1] yields the estimate

$$
\left|D_{\zeta}^{\alpha} D_{\bar{\zeta}}^{\beta} K_{\Omega^{\prime}}\left(\widetilde{\zeta}, \widetilde{\zeta}^{-}\right)\right| \lesssim \delta^{-2-\alpha_{2}-\beta_{2}} \tau\left(z^{\prime}, \delta\right)^{-2-\alpha_{1}-\beta_{1}}
$$

for any 2 -indices $\alpha, \beta$.

\section{Curvature estimates}

For the time being, $z^{\prime}$ will be fixed, and we will denote $K_{\Omega^{\prime}}$ by $K$ and derivatives of $K_{\Omega^{\prime}}$ with subscripts, e.g., $\left(\partial^{2} / \partial z_{1} \partial \bar{z}_{2}\right) K_{\Omega^{\prime}}=K_{1 \overline{2}}$. Set, for $1 \leq i$, $j \leq 2$ and $\zeta \in V$,

$$
g_{i j}(\zeta)=\frac{\partial^{2}}{\partial \zeta_{i} \partial \bar{\zeta}_{j}} \log K(\zeta, \bar{\zeta})
$$

Then, if $Y=c_{1} \partial / \partial \zeta_{1}+c_{2} \partial / \partial \zeta_{2}$, it is well known [1] that the Bergman length of $Y$ is given by

$$
B_{\Omega^{\prime}}(\zeta ; Y)=\left(\sum_{i, j=1}^{2} g_{i j}(\zeta) c_{i} \bar{c}_{j}\right)^{1 / 2} .
$$

If we compare coefficients in (6) and (8), we have

$$
\begin{aligned}
& \left|g_{11}(\tilde{\zeta})\right| \approx \tau\left(z^{\prime}, \delta\right)^{-2}, \quad\left|g_{22}(\tilde{\zeta})\right| \approx \delta^{-2}, \\
& \left|g_{12}(\tilde{\zeta})\right|=\left|g_{21}(\tilde{\zeta})\right| \approx \tau\left(z^{\prime}, \delta\right)^{-1} \delta^{-1} .
\end{aligned}
$$


Moreover, if we set $c_{2}=1$ and $c_{1}=-g_{22} / g_{12}$ in (6) and (8) and compare, we have

$$
\mid \operatorname{det}\left(g_{i j}(\widetilde{\zeta}) \mid \approx \tau\left(z^{\prime}, \delta\right)^{-2} \delta^{-2}\right.
$$

The components of the Riemann curvature tensor, $R^{\Omega^{\prime}}$, for the Bergman metric are locally defined by (11)

$$
\begin{aligned}
R_{i j k \bar{l}}^{\Omega^{\prime}}= & -\frac{\partial^{4}}{\partial \zeta_{i} \partial \bar{\zeta}_{j} \partial \zeta_{k} \partial \bar{\zeta}_{l}} \log K+\sum_{p, q=1}^{2} g^{p q} \frac{\partial^{3}}{\partial \zeta_{i} \partial \zeta_{k} \partial \bar{\zeta}_{p}} \log K \frac{\partial^{3}}{\partial \bar{\zeta}_{j} \partial \bar{\zeta}_{l} \partial \zeta_{q}} \log K \\
= & g_{i j} g_{k l}+g_{i l} g_{j k}-\overbrace{\frac{1}{K_{2}}\left(K \cdot K_{i \bar{j} k \bar{l}}-K_{i k} K_{\overline{j l}}\right)}^{P} \\
& +\frac{1}{K^{4}} \sum g^{p q}\left(K K_{i k \bar{p}}-K_{i k} K_{\bar{p}}\right)\left(K \cdot K_{\overline{j l} q}-K_{\overline{j l}} K_{q}\right) .
\end{aligned}
$$

We now estimate all the terms on the right hand side except the first two. When considering any term, list all the indices together and let $s$ denote the number of 1 and $\overline{1}$ appearing in the list and $t$ denote the number of 2 and $\overline{2}$ in the list. If $T$ denotes either of the terms in $P$, then (5) and (7) imply

$$
|T(\widetilde{\zeta})| \lesssim \tau\left(z^{\prime}, \delta\right)^{-s} \delta^{-t} .
$$

For the terms in the sum, note that (9) and (10) give

$$
\begin{aligned}
& \left|g^{11}(\tilde{\zeta})\right| \approx \tau\left(z^{\prime}, \delta\right)^{2}, \quad\left|g^{22}(\tilde{\zeta})\right| \approx \delta^{2}, \\
& \left|g^{21}(\tilde{\zeta})\right| \approx \tau\left(z^{\prime}, \delta\right) \delta \approx\left|g^{12}(\tilde{\zeta})\right| .
\end{aligned}
$$

Multiply the factors in the sum and let $T$ denote any of the resulting 16 terms; (5) and (7) show

$$
|T(\tilde{\zeta})| \lesssim\left|g^{p q}\right| \tau\left(z^{\prime}, \delta\right)^{-\tilde{s}} \delta^{-\tilde{t}}
$$

where $p$ and $q$ are included in the count for $\tilde{s}$ and $\tilde{t}$. Hence (12), gives

$$
|T(\widetilde{\zeta})| \lesssim \tau\left(z^{\prime}, \delta\right)^{-s} \delta^{-t}
$$

where $s$ and $t$ count only the indices of the component of the curvature tensor.

Let $Y=\sum_{i=1}^{2} b_{i} \partial / \partial \zeta_{i}$ be a holomorphic tangent vector with unit length,

$$
\sum g_{i j} b_{i} \bar{b}_{j}=1
$$

then the holomorphic sectional curvature determined by $Y$ is defined

$$
S(Y)=\sum R_{i j k \bar{l}}^{\Omega^{\prime}} b_{i} \bar{b}_{j} b_{k} \bar{b}_{l} \text {. }
$$

Note that (13) and the growth conditions (9) give

$$
\left|b_{1}(\tilde{\zeta})\right| \approx \tau\left(z^{\prime}, \delta\right), \quad\left|b_{2}(\tilde{\zeta})\right| \approx \delta
$$

unless $b_{i}(\tilde{\zeta})=0$. In either case, using $(11)$ and the estimates $(\mathrm{E})$ gives

$$
|S(Y)(\widetilde{\zeta})|=2+\mathscr{O}(1)
$$


where the constant is again independent of $\delta$ and $z^{\prime}$. To transfer this information to $\Omega$, recall that biholomorphisms are isometries in the Bergman metric, so, as tensors

$$
R^{\Omega}=\left(\Phi^{\prime-1}\right)^{*} R^{\Omega^{\prime}}
$$

This, if $X=\left(\Phi^{\prime}\right)_{*} Y$ and $z=\Phi^{\prime}(\tilde{\zeta})$ then

$$
|S(X)(z)| \leq C \text {. }
$$

Finally, we vary $z^{\prime}$ over a small boundary neighborhood of $z_{0}$ and allow $\delta$ to range in $\left(0, \delta_{0}\right)$. The set of points $z=\Phi^{\prime}(\tilde{\zeta})$ then form a neighborhood of the form $U \cap \Omega$ where $U$ is a neighborhood of $z_{0}$, and so the proof of the theorem is complete.

Remark. The estimates (E) give more control of the curvature tensor than we actually used. If we extend the domain of the Bergman metric to the set of all complex tangent vectors using the natural almost-complex structure map, computations as above give bounds on all the sectional curvatures near $z_{0}$.

\section{REFERENCES}

1. S. Bergman, The kernel function and conformal mapping, Amer. Math. Soc. Survey, Providence R.I., 2nd ed., 1970.

2. __ Sur la fonction-noyau d'un domaine..., Mem. Sci. Math. Paris 108 (1948).

3. J. Bland, The Einstein-Kähler metric on $\left\{|z|^{2}+|w|^{2 p}<1\right\}$, Michigan Math. J. 33 (1986), 209-220.

4. D. Catlin, Estimates of invariant metrics on pseudoconvex domains of dimension two, Math. Z., (to appear).

5. R. Greene and S. Krantz, Biholomorphic self-maps of domains, Lectures Notes in Math. vol. 1276, Springer-Verlag, pp. 136-206.

6. P. Klembeck, Kähler metrics of negative curvature ..., Indiana Math. J. 27 (1978), 275-282.

7. S. Kobayashi, The geometry of bounded domains, Trans. Amer. Math. Soc. (1959), 267-290.

8. A. Koranyi, A Schwarz lemma for bounded symmetric domains, Proc. Amer. Math. Soc. (1966), 210-213.

9. J. McNeal, Boundary behavior of the Bergman kernel function in $\mathbf{C}^{2}$, Duke Math. J., 58 (1989), 499-512.

10. N. Mok and S.-T. Yau, Completeness of the Kähler-Einstein metric and characterization of domains of holomorphy of curvature conditions, Proc. Symp. Pure Math. 39 (1983), 41-59.

11. S.-T. Yau, A general Schwarz lemma for Kähler manifolds, Amer. J. Math. 100 (1978), 197-203.

Department of Mathematics, Purdue University, West Lafayette, Indiana 47907 08544

Current address: Department of Mathematics, Princeton University, Princeton, New Jersey 